\title{
Automatic Multi-model-Based Segmentation of the Left Atrium in Cardiac MRI Scans
}

\author{
Dominik Kutra $^{1}$, Axel Saalbach ${ }^{1}$, Helko Lehmann ${ }^{1}$, Alexandra Groth ${ }^{1}$, \\ Sebastian P.M. Dries ${ }^{1}$, Martin W. Krueger ${ }^{2}$, Olaf Dössel ${ }^{2}$, and Jürgen Weese ${ }^{1}$ \\ 1 Philips Research Laboratories, Hamburg, Germany \\ 2 Institute of Biomedical Engineering, Karlsruhe Institute of Technology (KIT), \\ Karlsruhe, Germany
}

\begin{abstract}
Model-based segmentation approaches have been proven to produce very accurate segmentation results while simultaneously providing an anatomic labeling for the segmented structures. However, variations of the anatomy, as they are often encountered e.g. on the drainage pattern of the pulmonary veins to the left atrium, cannot be represented by a single model. Automatic model selection extends the model-based segmentation approach to handling significant variational anatomies without user interaction. Using models for the three most common anatomical variations of the left atrium, we propose a method that uses an estimation of the local fit of different models to select the best fitting model automatically. Our approach employs the support vector machine for the automatic model selection. The method was evaluated on 42 very accurate segmentations of MRI scans using three different models. The correct model was chosen in $88.1 \%$ of the cases. In a second experiment, reflecting average segmentation results, the model corresponding to the clinical classification was automatically found in $78.0 \%$ of the cases.
\end{abstract}

\section{Introduction}

With a prevalence of $0.4 \%$ to $1 \%$, atrial fibrillation (AF) is the most common cardiac arrhythmia in the USA and in Europe [1] and can lead to severe, lifethreatening conditions like stroke. Catheter ablation procedures aim at the electrical isolation of reentry pathways and ectopic foci that are causing AF in the atrial tissue. Triggering foci are often found in the pulmonary veins (PVs) of the left atrium (LA) [2]. The knowledge of the anatomy of the LA is crucial for successful procedures as it enables accurate planning of ablation lines and guidance during the procedure.

With the advent of ablation procedures to treat AF by the isolation of the $\mathrm{PVs}$, interest in the assessment of the PV configuration rose. Typically, the LA is joined by two PVs on each side through individual ostia: left superior and left inferior PV (LSPV, LIPV) on the left side of the LA; right superior and right inferior PV (RSPV, RIPV) on the right. This, typical pattern is found in about $60 \%$ of the patients [3. The most frequent variation on the left side of the LA is the common left trunk (CLT). Both left PVs merge into a single trunk in the 
proximity of the LA body. With a distance from the ostium to the bifurcation shorter than $1 \mathrm{~cm}$ to $1.5 \mathrm{~cm}$, depending on the study 34, it is called short CLT and occurs in $10 \%$ of the patients while the CLT is found in $4 \%$ to $8 \%$ of the patients. Kaseno et al. suggest a typical configuration as well as the short CLT to be normal [4. On the right side, the right middle PV pattern (RMPV), which is characterized by an accessory PV joining the LA body with a separate ostium, is found in $13 \%$ to $24 \%$ of the patients. The presence of concurrent variations on both sides of the LA is rarely reported.

Using model-based segmentation, very accurate segmentation results have been demonstrated in the past for the heart [567] in general and the LA in particular 8. However, using only a single model, variations in the anatomy of the LA cannot be reflected and thus, the segmentation is inaccurate. Different approaches emerged from the necessity to account for the variational anatomy while preserving the advantages of model-based segmentation. Zheng et al. presented a part based model approach, adapting the chamber with the LAA and the four major PVs as individual parts on C-arm CT datasets [9]. In the adapted state, the parts are joined, enabling the approach to represent both, two individual PVs on the left as well as the CLT pattern. Hanna et al. introduced a hybrid method combining model-based segmentation and guided region growing for the automatic detection of three PV patterns on the right side of the LA in CT images 10. This approach, however, is unsuitable for MRI because of image noise, lack of gray-level calibration, field inhomogeneities and artifacts due to patient movement. Incorporating anatomical variations into model-based segmentation remains a challenging and active area of research.

In our proposed approach, model-based segmentation as described in [11] is carried out with multiple models. We define a measure of the local segmentation fit in the areas where the models reflect the anatomical variations without the availability of ground truth annotations. This measure is then used as the input for a support vector machine (SVM) to automatically select the model resulting in the most accurate segmentation.

\section{Method}

\subsection{Used Image Database}

The used database consists of 59 whole heart (WH) scans of individual patients which were classified according to the anatomical variant by a clinical expert. About $53 \%$ of the atria had normal anatomy (typical or short CLT on the left and two PVs on the right), $14 \%$ the CLT and $25 \%$ the RMPV pattern. The scans were acquired on Philips Achieva $1.5 \mathrm{~T}$ systems at Kings College London using either contrast enhanced inversion recovery turbo field echo (IR-TFE) or steady state free precession (SSFP) protocols, both with SENSE encoding for shorter acquisition times. The same cardiac and breathing cycle was achieved using a navigator technique. The image resolution ranged from 0.72 to $1.48 \mathrm{~mm}$ in-plane and from 1.5 to $2.0 \mathrm{~mm}$ through-plane. 


\subsection{Shape Models of the Left Atrium}

To create models for the most frequent anatomical variations, $32 \mathrm{WH}$ scans were arbitrarily selected from the database: seven with typical anatomy, eleven with short CLT, seven with CLT and seven with RMPV pattern. We used the left atrium model described in [11] as the basis for the model generation. This model has two PVs on each side and was complemented by the trunk of the left atrial appendage (LAA). The model was manually adapted to one arbitrary dataset and an initial feature training was carried out to allow for coarse automatic segmentations of further datasets, which were manually refined to generate the ground truth. Training was carried out on 18 datasets, having either typical anatomy or short CLT on the left and two PVs on the right to represent the normal anatomy (normal model). To generate a model for the most frequent anatomical variation on the left side of the LA, the CLT, selective training on seven CLT datasets was performed (CLT model). For the RMPV model, a generic cylinder was manually merged to the original mesh, estimating its position, to account for the accessory PV joining the LA. Using this mesh in ground truth annotation for the RMPV-datasets and the following training led to the $R M P V$ model. The resulting mean meshes for all three models are depicted in Fig. 1 .

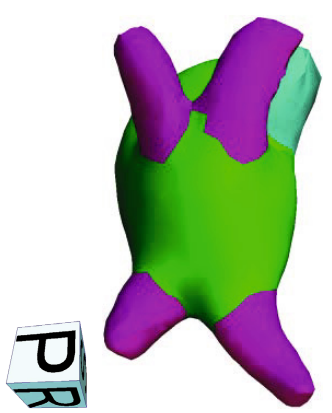

(a) normal model

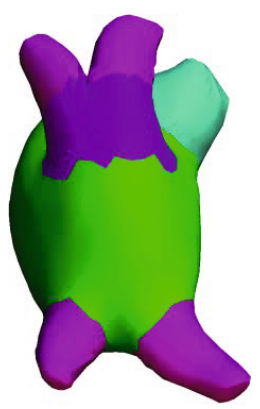

(b) CLT model

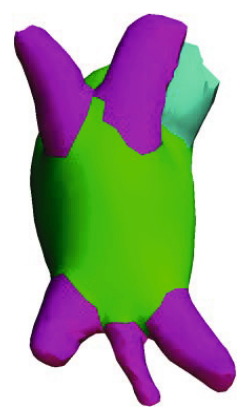

(c) RMPV model

Fig. 1. Resulting labeled mean meshes after training. The left atrial body is labeled green. All pulmonary veins are colored in violet. The LAA is colored in mint green. A special label was assigned to the CLT in dark violet (b).

\subsection{Segmentation Framework}

In order to generate a patient specific segmentation, consisting of a triangle mesh with labeled triangles, the automatic model-based segmentation framework described in [11] was used. In a nutshell, a hierarchical model of the organ with boundary detection functions trained on reference data is adapted to the 3-D image data. The attraction of the model to the image boundaries is realized by the search for target points, which is carried out for every triangle center along discrete positions on a search profile in the direction of the triangle normal. Target points are selected at the position having the largest weighted feature 
response, reflecting the reliability of the found image feature (eg. gray-level or gradient). The aim of the following adaptation steps is to successively minimize the distance between triangle centers and target points. In the beginning, the PVs and the LAA are inactive and only the LA body is adapted to the image. By applying a single similarity transform, the pose is corrected. Further adaptation is achieved by employing an affine transform to the whole mesh. Next, a multiaffine adaption is performed. To enhance segmentation accuracy, we assigned an extra transformation to the right side of the LA. In the last segmentation step a deformable adaption is performed to allow the triangle mesh to fully adapt to the patient's anatomy. In this step, the LAA and the PVs are successively activated and adapted to the image. Throughout the entire segmentation process, an external energy term is used that represents the image forces pulling the mesh towards image features. During deformable segmentation, the shape is constrained by an internal energy term which maintains the similarity to the original mean mesh.

For evaluation purposes, leave-one-out crossvalidation on the ground truth data was employed. Segmentation with the four chamber heart model, described in [11, previously trained on MRI, has been used to initialize the position of the LA. Symmetric constrained point-to-surface (CPS) distances between the automatically adapted meshes and the ground truth segmentations were computed according to [1]. The mean CPS distances $\epsilon_{\text {mean }}$ are shown in Tab. 1 . The overall CPS distance of about $1.1 \mathrm{~mm}$ for all models is in the range of the voxel resolution. Towards the more variable structures, like the LAA and the $\mathrm{PVs}$, the segmentation error rises but averages to less than $2 \mathrm{~mm}$. Compared to Ecabert et al. [11, who used CT-data with high resolution, the mean errors are larger. This difference is mainly accounted to the coarser resolution of the image data used in our work and the more challenging image characteristics of MRI.

Table 1. Mean constrained point-to-surface distances $\epsilon_{\text {mean }}[\mathrm{mm}]$ resulting from the leave-one-out crossvalidation for the three models

\begin{tabular}{lccccccccc}
\hline \multirow{2}{*}{ Model } & \multicolumn{8}{c}{$\epsilon_{\text {mean }}$ per region [mm] } \\
& LA body LSPV LIPV CLT RSPV RIPV RMPV LAA Whole mesh \\
\hline Normal & 0.87 & 1.56 & 1.81 & - & 1.41 & 1.11 & - & 1.70 & 1.14 \\
CLT & 0.81 & 1.17 & 1.73 & 1.18 & 1.17 & 1.16 & - & 1.25 & 1.01 \\
RMPV & 0.79 & 0.94 & 0.78 & - & 1.27 & 1.60 & 1.00 & 1.51 & 1.05 \\
\hline
\end{tabular}

\subsection{Automatic Model Discrimination}

To improve the model-based segmentation the three models have to be automatically selected for the respective datasets. The developed models possess differences only in certain regions, leading to different segmentation results in those regions on the same dataset. Information about the segmentation fit is extracted in these regions of interest (ROIs) (see Fig. 2) and used as input for the SVM. 
The classification problem is split into two binary classification sub-problems, testing each variant against the normal model. In the first sub-problem the left sides of the two models that are expected to result in different outcomes in the region of the left PVs are considered. Thus, the ROIs of the nomal model and the CLT model are chosen (Fig. $2 \mathrm{a}$ and $2 \mathrm{~b}$ ). Using the analogous argumentation for the right side of the models, the ROIs in the normal model and the $R M P V$ model, highlighted in Fig. $2 \mathrm{c}$ and $2 \mathrm{~d}$, are selected for the second sub-problem.

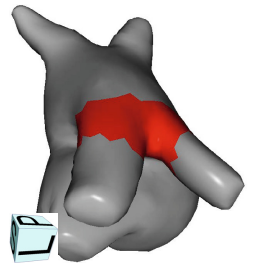

(a) normal model

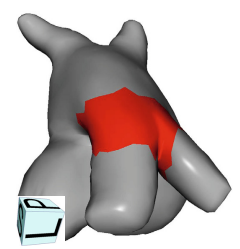

(b) CLT model

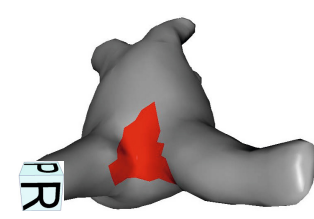

(c) normal model

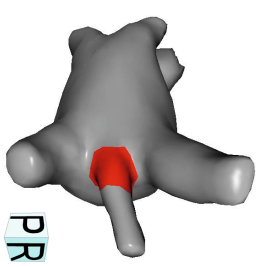

(d) RMPV model

Fig. 2. Regions of interest (ROIs) highlighted in red. For the first sub-problem, the regions in (a) and (b) are considered. For the second sub-problem, the regions in (c) and $(\mathrm{d})$ are selected.

Assessment of the segmentation fit exploits the fact that the model is only approximately adapted to the detected target points. To assess the segmentation fit, the search for target points is started again after the segmentation process is complete to get the distance to the target point and the corresponding feature response for each triangle center. We considered two approaches to construct the observation vectors for the SVM. Firstly, the vectors were constructed using spatial coding, ordered after the triangle index. Secondly, the ROIs were statistically analyzed and the resulting histogram bins were used as the elements of the observation vector. Considering different search profile lengths $(2,4$ and $10 \mathrm{~mm}$ ), we extracted 24 different kinds of SVM input vectors as candidates for our discriminative measure. As input data for the SVM, the histograms of the unsigned distances to the target point at a search profile length of $2 \mathrm{~mm}$ were found to perform the best.

For the training of the SVM, the complete image database of $59 \mathrm{WH}$ scans was considered to raise the number of available data and at the same time reducing the bias introduced on the datasets already used in the training of the models. All datasets were automatically segmented with the three models and selected as training data if one of the models yielded a very good segmentation result. For the annotated 32 datasets a mean CPS distance in the area of all ostia of less than $2 \mathrm{~mm}$ was considered to be very good. The automatic segmentation results for the remaining datasets were qualitatively evaluated and required to be similarly accurate in the regions of the ostia. In total, 42 datasets, of which 23 have been used in ground truth annotation, were selected for training of the SVM. To optimize the kernel-parameters for the Gaussian kernel, we performed the grid search approach with 5-fold crossvalidation according to 12 and adjusted the class weights to reflect the distribution of the PV-patterns in the image data. 


\section{Results}

\subsection{Discrimination Performance for Accurate Segmentations}

The aim of the first experiment was to evaluate the automatic model discrimination under optimal conditions. Leave-one-out crossvalidation was performed on the 42 very good training datasets. The class distribution of the datasets is as follows: $57 \%$ normal anatomy, $31 \%$ RMPV, $12 \%$ CLT pattern. For the first sub-problem in 40 of the 42 cases the model according to the clinical classification was selected. For the second sub-problem the correct model was chosen for 39 datasets $(92.86 \%)$. Combining the two sub-problems, the correct model was chosen for $88.1 \%$ of the datasets, meaning that only five of 42 datasets were falsely classified. The confusion matrix summarizes the classification results (Tab. 2). Examination of the automatically selected segmentations of the five datasets revealed that in one case, the automatically selected model (CLT model, yellow in Fig. 3a) yielded an absolutely comparable segmentation result, despite not matching the clinical classification (short CLT), as indicated by brackets in Tab. 2. Three datasets with a present third PV were not correctly classified by the SVM. In two of the cases the third PV has an early branching in the ROI of the RMPV model. In the other misclassified case the accessory PV has very low contrast. Taking into account that in one case the model chosen by the SVM yields a similar result, despite not matching the clinical classification, an accurate automatic segmentation is achieved for $90.5 \%$ of the datasets which means an increase of $33.5 \%$ compared to using a single model covering the majority class.

\subsection{Discrimination Performance for Average Segmentations}

We evaluated the model discrimination method in a second experiment with the 17 remaining datasets which were previously excluded because the automatic segmentation did not fulfill the criterion used to identify very accurate segmentations. It is known a priori that five of the datasets cannot be segmented satisfyingly with either of the models because their variants are not considered. Seven of the twelve remaining datasets can be segmented using the model of the majority class. In nine of the twelve cases, the SVM chose the model corresponding to the clinical classification for the first sub-problem, while only one dataset was falsely classified in the second sub-problem. The confusion matrix in Tab. 3 includes the results of the previous experiment to reflect the performance on average data. Summing it up, for the complete database, the model corresponding to the clinical classification was automatically chosen in $78.0 \%$ of the cases. As in the first experiment, we further examined the segmentation results of the misclassified cases. With the automatic model selection approach one dataset with a normal PV configuration was classified in the CLT class which resulted in an more accurate segmentation of the left PVs (see Fig. 3b). The RMPV was detected in one of two cases while simultaneously classifying the left side as CLT which yields a more accurate segmentation. In the other case the accessory $\mathrm{PV}$ is surrounded by structures that are not visible in the other datasets. 


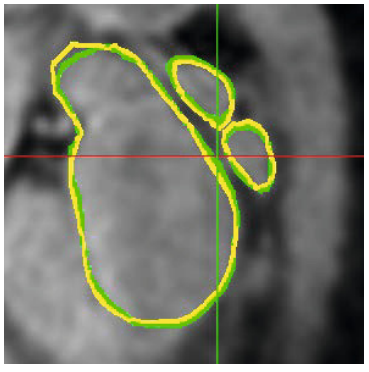

(a)

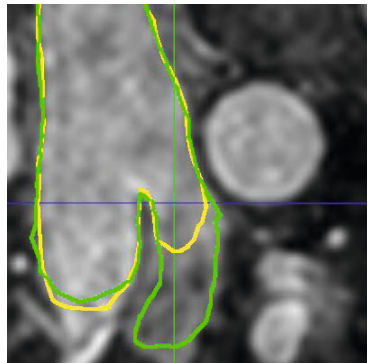

(b)

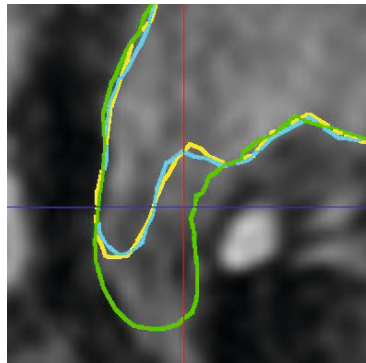

(c)

Fig. 3. Examples for successful selection of the better fitting model. Automatic segmentation results with the normal model is shown in yellow, model CLT is shown in green and model RMPV in blue.

Table 2. Confusion matrix for accurate segmentations. Values in brackets indicate false automatic classifications yielding an increased segmentation accuracy.

\begin{tabular}{|c|c|c|c|c|}
\hline Actual & \multicolumn{4}{|c|}{ Prediction } \\
\hline normal & 23 & (1) & 0 & 0 \\
\hline CLT & 1 & 4 & 0 & 0 \\
\hline RMPV & 3 & 0 & 10 & 0 \\
\hline other & - & - & - & - \\
\hline
\end{tabular}

Table 3. Confusion matrix for average segmentations

\begin{tabular}{|c|c|c|c|}
\hline \multicolumn{4}{|c|}{$\begin{array}{c}\text { Prediction } \\
\text { normal CLT RMPV CLT+RMPV }\end{array}$} \\
\hline 29 & (2) & 0 & 0 \\
\hline 2 & 6 & 0 & 0 \\
\hline 4 & 0 & 10 & (1) \\
\hline 2 & 1 & 2 & $\overline{0}$ \\
\hline
\end{tabular}

Especially in the region of the ROI, model RMPV does not yield an accurate segmentation. Considering the datasets that were classified falsely but result in a more accurate segmentation with the automatically selected model, 10 of the 12 additional datasets were segmented correctly. For the complete database an accurate segmentation has been achieved in $28.8 \%$ more of the cases compared to using only one model covering the majority class.

\section{Conclusion}

We present a new approach that enables multi-model-based segmentation by comparing the local fit of different adapted models and automatically choosing the best model using an SVM. Using the three created models, the method achieved a correct clinical classification in $88.1 \%$ of the cases and increased the fraction of datasets that could be accurately segmented from $57 \%$ to $90.5 \%$ under ideal conditions. If accurate segmentations are not achieved, the classifier performs less reliably as shown in the second experiment. Further improvements could be achieved by the generation of new models covering more variants. 
Acknowledgement. The research leading to these results has received funding from the European Community's Seventh Framework Programme (FP7/20072013) under grant agreement number 224495 (euHeart project). We would like to thank K. Rhode and R. Razavi for the acquisition of the image data.

\section{References}

1. Fuster, V., et al.: ACC/AHA/ESC 2006 guidelines for the management of patients with atrial fibrillation-executive summary. Circulation 114(7), 700-752 (2006)

2. Haïssaguerre, M., et al.: Spontaneous initiation of atrial fibrillation by ectopic beats originating in the pulmonary veins. N. Engl. J. Med. 339(10), 659-666 (1998)

3. Marom, E.M., et al.: Variations in pulmonary venous drainage to the left atrium: Implications for radiofrequency ablation. Radiology 230(3), 824-829 (2004)

4. Kaseno, K., et al.: Prevalence and characterization of pulmonary vein variants in patients with atrial fibrillation determined using 3-dimensional computed tomography. The American Journal of Cardiology 101(11), 1638-1642 (2008)

5. Ecabert, O., et al.: Automatic model-based segmentation of the heart in CT images. IEEE Trans. Med. Imag. 27(9), 1189-1201 (2008)

6. Peters, J., et al.: Optimizing boundary detection via simulated search with applications to multi-modal heart segmentation. Med. Image Anal. 14, 70-84 (2010)

7. Zheng, Y., et al.: Four-chamber heart modeling and automatic segmentation for 3D cardiac CT volumes using marginal space learning and steerable features. IEEE Trans. Med. Imag. 27(11), 1668-1681 (2008)

8. Manzke, R., et al.: Automatic segmentation of rotational X-ray images for anatomic intra-procedural surface generation in atrial fibrillation ablation procedures. IEEE Trans. Med. Imag. 29(2), 260-272 (2010)

9. Zheng, Y., Wang, T., John, M., Zhou, S.K., Boese, J., Comaniciu, D.: Multi-part Left Atrium Modeling and Segmentation in C-Arm CT Volumes for Atrial Fibrillation Ablation. In: Fichtinger, G., Martel, A., Peters, T. (eds.) MICCAI 2011, Part III. LNCS, vol. 6893, pp. 487-495. Springer, Heidelberg (2011)

10. Hanna, R., Barschdorf, H., Klinder, T., Weber, F.M., Krueger, M.W., Dössel, O., Lorenz, C.: A Hybrid Method for Automatic Anatomical Variant Detection and Segmentation. In: Metaxas, D.N., Axel, L. (eds.) FIMH 2011. LNCS, vol. 6666, pp. 333-340. Springer, Heidelberg (2011)

11. Ecabert, O., et al.: Segmentation of the heart and great vessels in CT images using a model-based adaption framework. Med. Image Anal. 15, 863-876 (2011)

12. Hsu, C.W., et al.: A practical guide to support vector classification. Department of Computer Science and Information Engineering, National Taiwan University, Taipei, Taiwan (2003) 